\title{
Selection for Resistance to Diamondback Moth (Plutella xylostella) in Cabbage
}

\author{
M.H. Dickson \\ Department of Horticultural Sciences, New York State Agricultural \\ Experiment Station, Cornell University, Geneva, NY 14456
}

\author{
A.M. Shelton and S.D. Eigenbrode \\ Department of Entomology, New York State Agricultural Experiment \\ Station, Cornell University, Geneva, NY 14456
}

\section{Margaret L. Vamosy and Marvin Mora \\ Panamerican School of Agriculture, Zamorano, Honduras}

Additional index words. insect resistance, breeding, Brassica oleracea var. capitata

\begin{abstract}
Inbreds and hybrid cabbage (Brassica oleracea var. capitata) selected for resistance to diamondback moth (DBM, Plutella xylostella L.) $_{\text {. }}$ were tested in the field in New York and Honduras for resistance. In New York, plants were inocrdated with up to 400 eggs per plant to enhance the severity of the tests. In Honduras, where natural populations of DBM were high, especially in the dry season, there were three distinct classes of susceptibility to DBM: the very susceptible controls or standard cultivars, the highly resistant glossy-leaved lines, and the intermediate selections with normal leaf bloom. Some normal-leaf hybrids were more resistant than either of their parents, which indicates the need to select for specific combining ability for high resistance levels. At maturity, the glossy-leaved hybrids produced marketable crops with: out aid of chemical sprays.
\end{abstract}

The diamondback moth (DBM) is the major limiting factor in crucifer production in tropical areas of the world. In the Philippines and Southeast Asia, Central and South America, as well as in the Caribbean Basin, the current strategy for controlling DBM with insecticides is failing because of resistance to insecticide (Talekar and Griggs, 1986). Such resistance is also becoming widepsread in the United States, as evidenced in a survey of 40 DBM populations collected and bioassayed in 1988-89 (Shelton and Wyman, unpublished data). Because of resistance to insecticide and insecticide residues on food crops and in the environment, research is needed to develop new control strategies that are more environmentally sound and more durable. Promising approaches include the development of resistant crop cultivars (Dickson et al., 1986).

In 1972 (Dickson and Eckenrode, 1975), we identified PI 234599, a glossy-leaved cauliflower, as being highly resistant in the field to the imported cabbage worm (ICW) (Pieris rapae L.) and the cabbage looper (CL) [Trichoplusia ni (Hubner)]. In 1978, we found it was, also resistant to DBM in Australia and the United States (Dickson and Eckenrode, 1980). Genetic studies showed the resistance was linked to the glossy dark-green leaf found

Received for publication 1 Nov. 1989. The cost of publishing this paper was defrayed in part by the payment of page charges. Under postal regulations, this paper therefore must be hereby marked advertisernent solely to indicate this fact. in PI 234599. In $\mathrm{F}_{2}$ populations, there was some variation among glossy plants for the level of resistance, but all were moderately or very resistant, while nonglossy plants were moderately resistant or very susceptible (Dickson and Eckenrode, 1980). Furthermore, we observed that when glossy selections were crossed with nonglossy tolerant selections, the $F_{1}$ was often more susceptible than a cross between two nonglossy lines with intermediate insect resistance. We concluded that this was the case because the glossy-leaf resistance masked other genes for resistance or susceptibility. In addition to the resistance in the glossy leaf, there was also evidence of a preference for oviposition (Jure et al., 1984) on glossy-leaved lines in the greenhouse, although this preference was not evident in field studies (Shelton et al., 1988).

Most of the above studies were done with natural field populations of lepidopterous pests, and levels of resistance were based on number of eggs, larvae, and/or pupae observed or on damage levels. While these studies have been useful, it was recognized that in small-plot evaluations, small differences in ovipositional preferences may cause large differences in larval counts and plant injury, but in a "nonchoice" situation, such as a commercial field, such differences may not occur (Cantelo and Sanford, 1984; Shelton et al., 1988). To overcome this problem, we began to inoculate field-grown plants in 1985 with known numbers of eggs of DBM, $\mathrm{CL}$, or ICW and then evaluate the survivorship and plant injury. The threshold recom- mended for spraying fresh-market cabbage in New York state to control DBM is 10 larvae/plant. Because resistance to the three insect species was similar and because DBM was a more important pest on a worldwide basis, we inoculated plants only with eggs of DBM beginning in 1986.

In this study, we report the results of screening inbreds and hybrids in the field in New York state using a procedure of placing laboratory-reared DBM eggs directly on each plant to be tested and then assessing the injury. These results are compared to tests conducted in Honduras using the same plant lines, but tested under severe natural infestations.

New York trials. In 1986,52 cabbage lines that had been selected for ICW and CL resistance in the field were screened for DBM resistance. The selections were developed from the cross of PI 234599 cauliflower to cabbage, followed by selection, backcrossing, recombining, and reselection. Resistance was determined by rating the plants for injury on a scale of 1 (no damage) to 5 (severe damage) $\approx 3$ weeks after the eggs were placed on the plants. Based on these results, selections were made, inbreds increased, and hybrids developed. These $F_{1}$ hybrids were screened in the field in 1987 (two replicates) and 1988 (four replicates), both hot, dry seasons conducive to high DBM population.

Plantings in both years consisted of a randomized complete-block design with 10 plants per plot. Plants were spaced in rows $0.90 \mathrm{~m}$ apart with a between-plant spacing of 0.45 $\mathrm{m}$. An insecticide was applied during transplanting for maggot control, but no further insecticides were applied. Three to five plants per plot were inoculated with DBM eggs. These eggs, laid on aluminum foil, were obtained from laboratory colonies established from insects collected annually in the field near Geneva, N. Y., and maintained on an

Table 1. DBM damage scores in cabbage parents and hybrids in 1987 and 1988 in New York.

$1=$ No damage, $5=$ severe damage. 'Glossy leaf. 

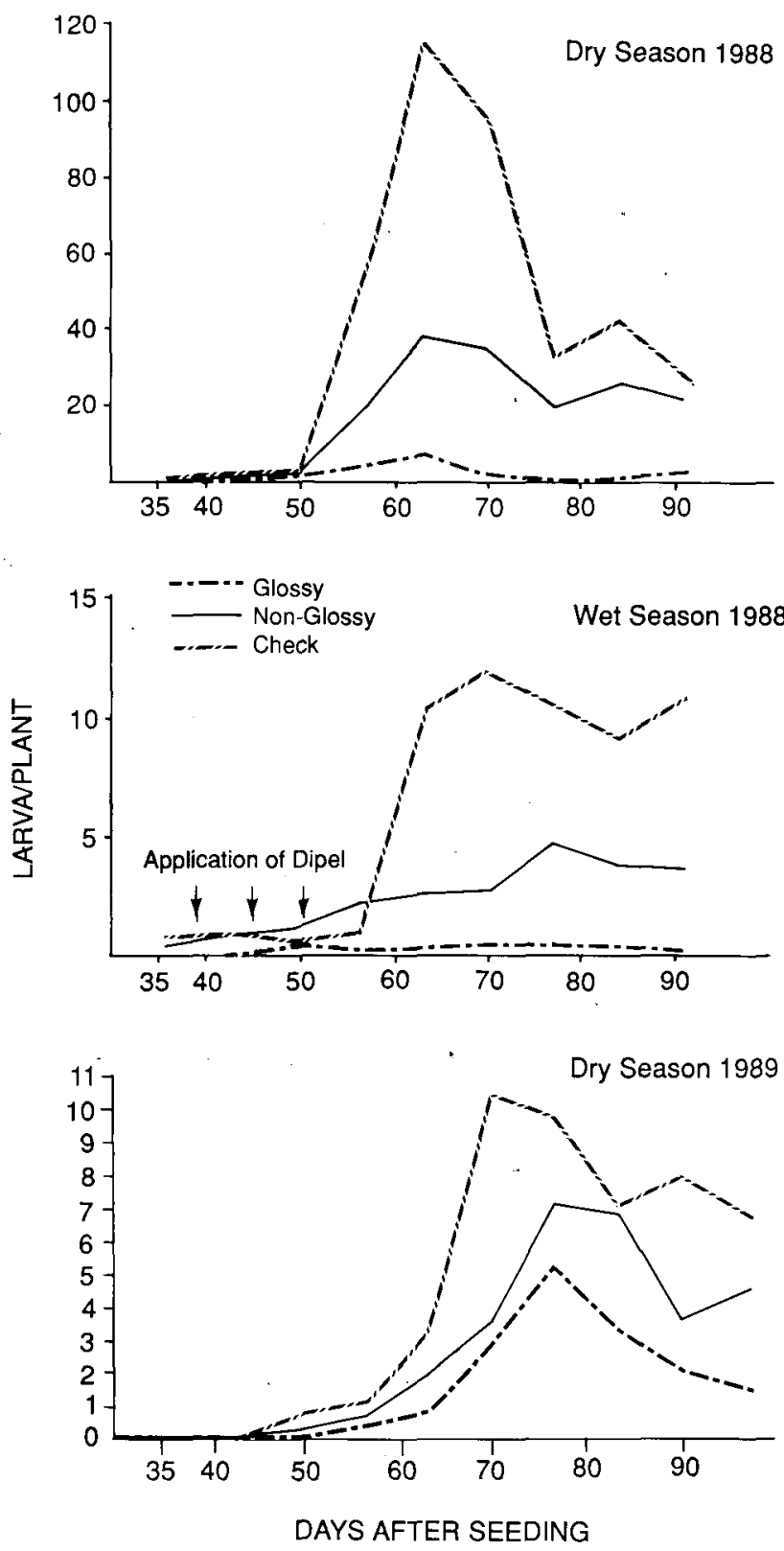

Fig. 1. Larval counts in the three seasons in Honduras for susceptible cultivars, tolerant lines with normal leaf bloom, and three resistant glossy-leaved lines of cabbage.

artificial diet (Beaver and Boldt, 1971). For antibiosis tests, $400 \mathrm{DBM}$ eggs per plant were placed o 6-week-old plants. Plants at least 6 weeks old were used because it was previously observed that resistance in some lines was not fully expressed until plants were 40 to 50 days old (Dickson and Eckenrode, 1980).

Honduras trials. The cabbage lines were planted in three, separate trials in Honduras at the Panamerican Agricultural School, El Zamorano: The first planting was seeded 25 Jan. 1988; the second, 7 Mar. 1988; and the third, 25 Jan. 1989. The January seedings produced crops during the dry season, when DBM incidence and damage are greatest; the March seeding compared performance of the cabbages under rainy-season conditions.

In all three trials, seedlings were transplanted to rows spaced $0.75 \mathrm{~m}$ apart with a between-plant spacing of $0.4 \mathrm{~m}$. Randomized complete blocks with three replicates were used in all three plantings. The 1988 dry-season trials included six lines and three commercial cultivar checks, with nine plants per plot; the 1988 rainy-season trial included 14 hybrids and three commercial cultivars, with seven plants per plot; and the 1989 dryseason trial included five lines and two commercial cultivars, with 40 plants per plot.

No insecticide applications were made to either of the dry-season plantings; Bacillus thuringiensis was applied three times, at 38 , 44 , and 50 days after seeding, at the start of the rainy-season trial to prevent early damage to the growing points. Low initial DBM populations in the 1989 trial made such applacations unnecessary.

Seed was supplied for these trials based on previous performance in trials in New York and on available seed supply. Thus, the 1988 Honduras trials were based on 1987 New York trials and available remnant seed, and the 1989 Honduras trials were based on 1988 trials and remnant seed.

Larval counts were made every 7 days, starting 42 days after seeding, on all nine plants per plot in the Jan. 1988 trial, on all seven plants per plot in the Mar. 1988 trial, and on 10 of the 40 plants per plot in the Jan. 1989 trial. Damage assessments were made twice in each trial, at 75 and 95 days after seeding in the Jan. 1988 trial and at 70 and 98 days after seeding in both the Mar. 1988 and Jan. 1989 trials.

Mean larval counts at each sampling date and damage scores at both rating times were compared by analysis of variance, and LSD values were computed.

New York trials. The glossy-leaved line had a lower damage rating (usually varying from 1 to 2) than any of the normal-bloomed cabbage (Table 1). However, some of the normal-leaved hybrids were significantly less damaged than the control (Roundup). All of the experimental hybrids are from New York inbreds.

Some hybrid lines, such as 2607 x 2624 and $2607 \times 2535$, were more resistant than either parent, although significantly more for only one parent (Table 1). Line 2537 also combined well to produce some attractive and resistant hybrids. Likewise, the crosses of 2592, 2542, and 2535 in all combinations showed significant resistance, as they had in 1986 in a severe ICW trial.

Honduras trials. The 1988 dry-season planting was subject to intense pressure of a very high natural DBM population, with larval counts reacing $>95$ per plant in all three commercial cultivars used as controls. During the 1988 rainy season, larval counts were only 10 per plant in the commercial cultivars. In the 1989 dry season, larval counts were similar to those of the rainy season and much lower than those of the previous dry season, presumably due to lower temperatures in 1989 than in the 1988 dry season. Nevertheless, in all three trials, there were significant differences in damage scores per plant between the commercial cultivars, the nonglossy lines, and the glossy-leaved hybrids (Table 2 ).

Figures 1 and 2 illustrate the larval count of the three groups of lines tested during the three trial periods. The larval counts and the damage ratings were highest in the 1988 dry season, but differences between commercial cultivar controls, nonglossy lines, and glossyleaved lines were evident in all three trials; glossy-leaved lines were the most resistant, nonglossy lines had intermediate resistance, and commercial cultivars had the least resistance to DBM.

Figure 2 shows the fluctuation in the number of larvae per plant over the season for individual lines of the three types of cabbage. However, genetic differences kept them separated into three classes of resistancesusceptible, intermediate, and resistantthroughout the season.

Table 2 and Fig. 1 can be used to compare 
Table 2. DBM damage scores in cabbage during three seasons in Honduras.

\begin{tabular}{|c|c|c|c|c|c|c|}
\hline \multirow[b]{3}{*}{ Pedigree } & \multicolumn{6}{|c|}{ Damage ratings $^{z}$} \\
\hline & \multicolumn{2}{|c|}{ Dry season (1988) } & \multicolumn{2}{|c|}{ Rainy season (1988) } & \multicolumn{2}{|c|}{ Dry season (1989) } \\
\hline & 75 days & 95 days & 70 days & 98 days & 70 days & 98 days \\
\hline Green Boy (control) & 5.0 & 4.0 & 3.3 & 4.9 & 2.1 & 3.6 \\
\hline Izalco (control) & 4.8 & 3.2 & 3.4 & 4.8 & 1.8 & 3.1 \\
\hline Bravo (control) & 5.0 & 5.0 & 3.4 & 5.0 & & \\
\hline 2506 & 4.6 & 3.3 & 2.3 & 3.6 & & \\
\hline 2623 & 4.3 & 5.0 & 2.0 & $3.3^{\circ}$ & & \\
\hline 2542 & 4.6 & 3.7 & 2.0 & 3.5 & & \\
\hline $2518 \times 2519 \mathrm{~F}_{1}^{\mathrm{y}}$ & 2.1 & 1.3 & 1.2 & 1.7 & & \\
\hline $2519 \times 2525 \mathrm{~F}_{1}^{\mathrm{y}}$ & 2.4 & 1.3 & 1.1 & 1.8 & & \\
\hline $2519 \times 2521 \mathrm{~F}_{1}^{\mathrm{y}}$ & 3.0 & 2.3 & 1.2 & 1.9 & & \\
\hline 2535 & & & 3.2 & 3.3 & & \\
\hline $1365 \times 1353 \mathrm{~F}_{1}$ & & & 3.1 & 3.7 & & \\
\hline $2542 \times 2525 F_{1}$ & & & 3.7 & 3.1 & & \\
\hline $2592 \times 2590 \mathrm{~F}_{1}$ & & & 2.6 & 4.2 & & \\
\hline $2542 \times 2535 \cdot F_{2}$ & & & 3.0 & 3.6 & & \\
\hline $2592 \times 2535 \mathrm{~F}_{3}$ & & & 3.0 & 2.8 & & \\
\hline $2592 \times 2542 \mathrm{~F}_{3}$ & & & 2.6 & 4.5 & & \\
\hline $2532 \times 9602 \mathrm{~F}_{1}^{\mathrm{y}}$ & & & & & 1.90 & 2.8 \\
\hline $2518 \times 2528 \mathrm{~F}_{1}^{\mathrm{y}}$ & & & & & 1.93 & 3.3 \\
\hline $2537 \times 2592 \mathrm{~F}_{1}$ & & & & & 1.96 & 3.0 \\
\hline $2607 \times 2624 \mathrm{~F}_{1}$ & & & & & 1.60 & 3.3 \\
\hline $2607 \times 2535 \mathrm{~F}_{1}$ & & & & & 1.56 & 2.9 \\
\hline Significance & $* *$ & & $* *$ & $* *$ & $* *$ & NS \\
\hline LSD,$P=0.05$ & 0.64 & & 1.20 & 1.34 & 0.26 & \\
\hline Coefficient of variation & $6.71 \%$ & & . 21.01 & 17.24 & 5.70 & \\
\hline
\end{tabular}

${ }^{\mathrm{z}} 1$ = No damage, 5 = severe damage.

${ }^{y}$ Glossy leaf.

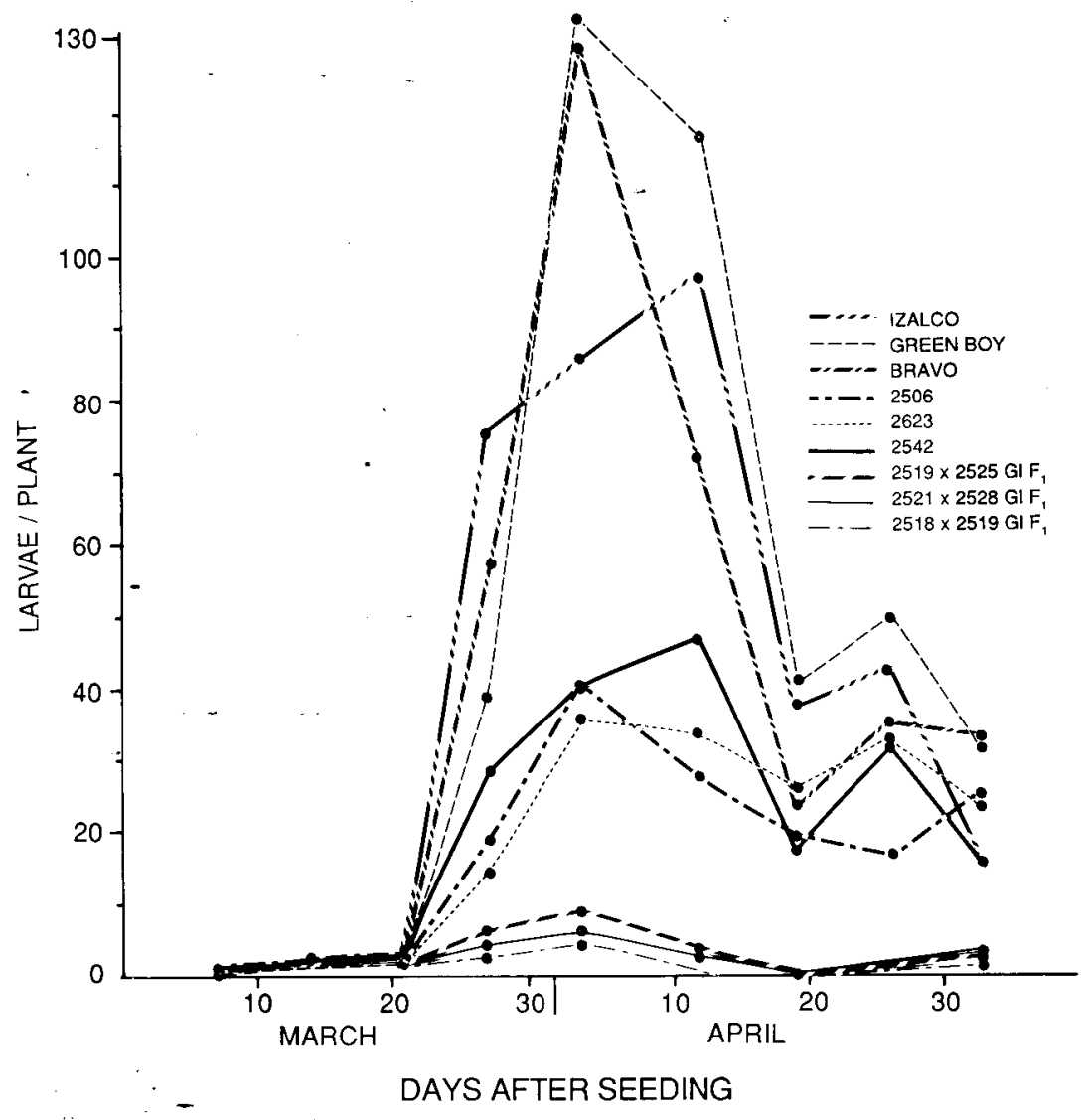

Fig. 2. Larval counts in the 1988 dry season in Honduras for three susceptible cultivars, three tolerant lines with normal leaf bloom, and three resistant glossy-leaved lines of cabbage.

damage scores and DBM counts for the 1988 dry season in Honduras. In the dry season, larval counts for 2506 and 2542 were always less than for the susceptible control, and the damage rating was significantly less, al- though still considerable. Both damage scores and larval count were low for the glossy hybrids. In the 1988 wet season, the larval counts were much lower than for the dry season, but the damage scores were similar. The very high larval count in the dry season resulted in more damage, but not proportionally to the increased larval counts. The tolerant plants had both fewer larvae and less damage, even in the dry season.

Glossy-leaved cabbage displayed higher levels of resistance to DBM in both the artificial inoculations done in New York and under the extreme natural infestations in Honduras. When we started this program in 1970, the glossy-leaved plants were not widely accepted for fresh-market use because they are dark green. With the change in attitude toward the use of chemicals and their effect on the environment, glossy-leaved cabbage is now being received favorably. In areas such as Honduras or tropical Asia, where there are high DBM populations that cannot be controlled by insecticides or where cabbage production otherwise might not be economically feasible, their use has become more acceptable, particularly if the outer leaves are removed. Some early damage, even to the glossy-leaved plants, has been observed, especially in Honduras and the Philippines (Dickson and Eckenrode, 1980).

We observed that not all glossy lines are highly resistant with severe DBM selection pressure. This is apparent in the 1989 dryseason results (Table 2, Fig. 1), where two glossy hybrids, $2532 \times 9602$ and $2518 \times 2528$, were not as resistant or low in larval numbers as some hybrids tested in the two previous seasons, although they had looked very resistant in our 1986 trial in New York. Thus, early season protection may be necessary, but insecticide costs with glossy-leaved cabbage should be greatly reduced compared to those with commercial cultivars, and insecticide would not be needed during the 30 to 40 days before harvest.

Even though resistance in selections with normal bloom is not as good as those with glossy leaves, it can result in significantly less damage in some hybrids. This result was encouraging, as higher levels of resistance than previously observed were found in plants with normal leaf bloom. These results indicate the importance of combining the right parents to obtain maximum resistance.

Since the level of resistance in the hybrids with normal leaves is only partial, trials will be needed in combination with integrated pest management to test if the number of sprays needed to achieve undamaged crops can be reduced. For less critical markets and lowinput production, these hybrids may offer economic resistance, especially in more temperate climates.

The first experimental hybrids (1987 and earlier years) were selected primarily for insect resistance, and many were weak and late. However, subsequent selection has also been for vigor and earliness. The enhanced resistance of some hybrids is not related to plant vigor or size in these trials because DBM egg populations were high enough in all experiments to cause severe injury to all nonresistant plants. In selecting for DBM resistance in broccoli (unpublished data), we have observed that DBM on resistant plants will remain on the inoculated leaf and pri- 
marily around the site of inoculation; DBM will spread all over a susceptible plant.

Although DBM resistance was the primary aim, selection was also made for ICW resistance at plant maturity. The resistance for both was generally similar (unpublished data), but in tests at maturity, damage due to ICW may be more apparent than that due. to DBM.

\section{Literature Cited}

Beaver, K.D. and P.E. Boldt. 1971. Continuous laboratory rearing of the diamondback moth and related biological data. Entomol. Soc. Amer. 64:651-655.
Cantelo, W.W. and L.L, Sanford. 1984. Insect population response to mixed and uniform plantings of resistant and susceptible plant material. Environ. Entomol. 13: 1443-1445.

Dickson, M.H. and C.J. Eckenrode. 1975. Variation in Brassica oleracea resistance to cabbage looper and imported cabbage worm in the greenhouse and field. J. Econ. Entomol. 98:757760 .

Dickson, M.H. and C.J. Eckenrode. 1980. Breeding for resistance in cabbage and cauliflower to cabbage looper, imported cabbage worm and diamondback moth. J. Amer. Soc. Hort. Sci 105:782-785.

Dickson, M. H., C.J. Eckenrode, and J. Lin. 1986. Breeding for diamondback moth resistance in
Brassica oleracea. First Intl. Diamondback Moth Wrks. Asian Vegetable Res. \& Dev. Ctr., Shanhua, Taiwan. p. 137-143.

Juru, Lin, M.H. Dickson, and C.J. Eckenrode. 1984. Resistance of Brassica lines to the diamondback moth (Lepidoptera: Yponomeutidae) in the field, and inheritance of resistance. J. Econ. Entomol. 77:1293-1296.

Shelton, A. M., C.W. Hey, R.C. North, M.H Dickson, and J. Barnard. 1988. Analysis of resistance in cabbage varieties to damage by Lepidoptera and Thysanoptera. J. Econ. Entomol. 81:634-640.

Talekar, N.S. and T.D. Griggs. 1986. Diamondback moth management. Proc. First Intl. Wrks. AVRDC, Shanhua, Taiwan. p. 9-10. 